\title{
Nilpotente Liesche Gruppen haben symmetrische Gruppenalgebren
}

\author{
Detlev Poguntke \\ Fakultät für Mathematik der Universität, Postfach 8640 , D-4800 Bielefeld, Bundesrepublik Deutsch- \\ land
}

Eine involutive (Banachsche) Algebra $A$ heißt symmetrisch, wenn jedes Element der Form $x^{*} x, x \in A$, ein positives Spektrum hat. Für Banachsche Algebren ist diese Eigenschaft nach einem Satz von Shirali und Ford äquivalent zu der Tatsache, daß hermite'sche Elemente ein reelles Spektrum haben, vgl. [2]. Über die Bedeutung der Symmetrie, insbesondere für die Darstellungstheorie, sei hier nichts gesagt, vgl. dazu [12] und [13]. Dort findet man auch eine Zusammenstellung der vorhandenen Ergebnisse über die Symmetrie bzw. Nicht-Symmetrie von $L^{1}$-Algebren lokalkompakter Gruppen $G$. Einige davon seien hier kurz wiedergegeben. Für eine zusammenhängende nicht-kompakte halbeinfache Liesche Gruppe $G$ ist $L^{1}(G)$ nicht symmetrisch, [6]. Aber $L^{1}(G)$ ist symmetrisch, falls $G$ eine diskrete, endlicherzeugte, nilpotente Gruppe ist, [5], $G$ eine Bewegungsgruppe, d. h. ein semidirektes Produkt einer kompakten Gruppe mit einem abelschen Normalteiler ist, [4], $G$ eine $[F C]^{-}$-Gruppe ist, d.h. falls $\left\{y x y^{-1} ; y \in G\right\}^{-}$für jedes $x \in G$ kompakt ist, [1], $G$ eine zusammenhängende nilpotente Liesche Gruppe der Stufe 2 ist, [10].

Weiter ist die Klasse $[S]$ der lokalkompakten Gruppen $G$ mit symmetrischen Gruppen-Algebren stabil gegen endliche Erweiterungen und gegen zerfallende kompakte abelsche Erweiterungen, d.h. mit $M$ liegt auch $K \ltimes M$ in [S] für kompakte abelsche Gruppen $K,[12]$. In [13] hat Leptin gezeigt, daß gewisse semidirekte Produkte zusammenhängender abelscher Gruppen in [S] liegen, insbesondere die , $a x+b$ "-Gruppe. Diese Gruppen waren sämtlich vom Typ I, aber ich werde in einer folgenden Arbeit zeigen, daß auch die „kleinste” auflösbare, zusammenhängende Liesche Gruppe, welche nicht vom Typ I ist, nämlich die „Mautner-Gruppe", in [S] liegt, wodurch die in [13] geäußerte Vermutung, daß alle zusammenhängenden auflösbaren Lieschen Gruppen in [S] liegen, erhärtet wird. Das Ziel der vorliegenden Arbeit ist es jedoch, den in der Überschrift angegebenen Satz zu beweisen, unten als Satz 2 formuliert. Zuvor beweisen wir noch ein Lemma und Satz 1. Das Lemma ist eine ziemlich einfache Verallgemeinerung von Satz 2 aus [12] und enthält ein auf die Anwendung im Beweis von Satz 1 zugeschnittenes Kriterium für Symmetrie; den Beweis in [12] kann man nahezu Wort für Wort übernehmen. Satz 1 enthält den entscheidenden Schritt auf dem Wege zum Beweis der Symmetrie nilpotenter Liescher Gruppen. Obwohl ich bislang im wesentlichen 
nur eine Anwendung von Satz 1 kenne, nämlich auf den nilpotenten Fall, habe ich die unten angegebene allgemeinere Formulierung von Satz 1 gewählt, da Aussicht besteht, unter Verwendung dieses Satzes zu zeigen, daß zusammenhängende auflösbare Liesche Gruppen vom Typ $E$ in $[S]$ liegen. Zum Beispiel kann man mit Satz 1 zeigen, daß eine Gruppe minimaler Dimension unter den nichtsymmetrischen, zusammenhängenden auflösbaren Lieschen Gruppen vom Typ E notwendigerweise ein eindimensionales Zentrum besitzt (natürlich nur, sofern es solche Gruppen überhaupt gibt).

Zunächst sei kurz an den Begriff der adjungierten Algebra erinnert, vgl. [7] und [8]. Dazu sei $A$ eine involutive Banachsche Algebra, welche der Bedingung $\left(L^{*}\right)$ aus [7] genügt, d. h. für alle $x \in A$ gelte

$$
\|x\|=\sup _{\|y\| \leqq 1}\|x y\|
$$

( = $\left\|L_{x}\right\|$, wenn $L_{x}: A \rightarrow A$ durch $w \rightarrow x w$ definiert ist). Offenkundig ist ( $\left.L^{*}\right)$ von selbst erfüllt, wenn es in $A$ eine beschränkte approximierende Eins gibt, d. h. ein Netz $\left(e_{\lambda}\right)$ mit $\left\|e_{\lambda}\right\|=1$ und $\lim _{\lambda} x e_{\lambda}=\lim e_{\lambda} x=x$ für alle $x \in A$. Dann ist die Menge aller beschränkten linearen Operatoren $T: A \rightarrow A$, für welche ein (eindeutig bestimmter) beschränkter linearer Operator $T^{*}: A \rightarrow A$ mit $(T x)^{*} y=x^{*} T^{*} y$ für $x, y \in A$ existiert, in der Operator-Norm eine involutive (mit Involution $T \rightarrow T^{*}$ ) Banachsche Algebra; diese wird mit $A^{b}$ bezeichnet. Weiter ist $x \rightarrow L_{x}$ ein isometrischer *Isomorphismus von $A$ auf ein zweiseitiges abgeschlossenes Ideal in $A^{b} ; A$ läßt sich mithin mit einem Ideal in $A^{b}$ identifizieren, und wir werden im folgenden diese Identifikation ohne weiteren Kommentar benïtzen. Es sei noch bemerkt, daß $L^{1}(G)^{b}$ für eine lokal-kompakte Gruppe $G$ nichts anderes als die Maßalgebra von $G$ ist.

Lemma. Es sei B eine involutive Banachsche Algebra mit beschränkter approximierender Eins. Ferner sei 2 eine Menge von Projektoren (d.h.q $=q^{*}=q^{2}$ für $q \in \mathbb{Q}$ ) in $B^{b} . Z u$ jedem $(p, q) \in \mathscr{Q} \times \mathscr{Q}$ sei ein $v_{p q} \in B^{b}$ gegeben, und es gelte

(i) $v_{p q}^{*}=v_{q p^{*}}$

(ii) $v_{p q} q=v_{p q}$ (und dann auch, wegen (i), $v_{q p}=q v_{q p}$ ).

(iii) $p=v_{p q} v_{q p}=v_{q p}^{*} v_{q p}$ für alle $p, q \in 2$.

(iv) Zu einer endlichen Teilmenge $\mathscr{E}$ von $\mathscr{Q}$ undeinem ausgezeichneten Element $q \in \mathscr{E}$ mögen stets $q_{1}, \ldots, q_{n} \in 2$ existieren mit $q_{1}=q, q_{i} q_{j}=\delta_{i j} q_{i}(1 \leqq i, j \leqq n)$ und derart, daß $\mathscr{E}$ in dem von $q_{i}$ und $v_{q, q}(1 \leqq i, j \leqq n)$ aufgespannten linearen Teilraum liegt.

Weiter liege $\sum_{p, q \in 2} p B q$ dicht in $B$.

Ist dann $p B p$ symmetrisch für ein $p \in \mathscr{Q}$ (und mithin für alle), so ist $B$ symmetrisch.

Beweis. Zunächst sei bemerkt, daß für $p, q \in \mathscr{Q}$ die Beziehung $(p B q q B q)^{-}=p B q$ gilt. Die eine Inklusion ist trivial ( $p B q$ ist abgeschlossen!). Seien nun $x \in p B q$ und $\left(e_{\alpha}\right)$ eine approximierende Eins in $B$. Dann ist $x e_{\alpha} q \in p B q B q$ und $\lim _{\alpha} x e_{\alpha} q=x$.

Um die Symmetrie von $B \mathrm{zu}$ beweisen, verwenden wir die in (2) aus [12] gegebene Charakterisierung. Sei also $L$ ein maximales modulares Linksideal in $B$. 
Wir haben die Existenz einer nicht-trivialen stetigen hermite'schen positiv definiten Linearform $f$ auf $B$ mit $f(L)=0$ zu zeigen. Wegen der Dichtheit von $\sum_{p, q \in E} p B q$ gibt es $p, q \in 2$ mit $p B q \not L$. Dann ist aber auch $q B q \not \subset L$. Wenn nicht, so gälte $(p B q)(q B q) \subset L$ und mithin $L \supset(p B q q B q)^{-}=p B q$.

$L_{0}:=q B q \cap L$ ist ein echtes Linksideal in $q B q$. Weiter gilt $B=B q+L$, und es gibt folglich eine Rechtseins $e$ in $B q$ für $L$, also $e q=e$. Dann ist $q e q=q e$ eine Rechtseins für das Linksideal $L_{0}$ in $q B q . L_{0}$ ist also ein modulares Linksideal in der symmetrischen Algebra $q B q$, und es gibt mithin ein nicht-triviales, stetiges, hermite'sches, positiv definites Funktional $f_{0}$ auf $q B q$ mit $f_{0}\left(L_{0}\right)=0$.

Für $x \in B$ setzen wir ( $e^{*} B e$ liegt in $q B q$ !)

$$
f(x)=f_{0}\left(e^{*} x e\right)
$$

und behaupten, daß dieses $f$ unser Problem löst.

Zunächst zeigen wir, daß $f_{0}=\left.f\right|_{q B q}$; insbesondere ist dann $f \neq 0$. Für $x \in q B q$ ist $x-x e \in L \cap q B q=L_{0}$, mithin $f_{0}(x-x e)=0$ oder $f_{0}(x)=f_{0}(x e)$. Da $f_{0}$ hermite'sch ist, gilt weiter $f_{0}(x)=f_{0}\left(x^{*}\right)^{-}=f_{0}\left(x^{*} e\right)^{-}=f_{0}\left(e^{*} x\right)$, also $f_{0}(x)=f_{0}(x e)=f_{0}\left(e^{*} x\right)$ $=f_{0}\left(e^{*} x e\right)=f(x)$. Es gilt $f(L)=0$; denn ist $x \in L$, so auch $x e$ (wegen $\left.x \equiv x e \bmod L\right)$, und letztlich $e^{*} x e \in L$, also in $L_{0}$. Daher ist $0=f_{0}\left(e^{*} x e\right)=f(x)$. Offenkundig ist $f$ hermite'sch. Wir müssen nur noch die Positivität von $f$ zeigen. Wegen der Stetigkeit von $f$ genügt es zu beweisen, daß $f\left(x^{*} x\right) \geqq 0$ für $x \in \sum_{p, r \in \mathscr{Q}} p B r$. In der SummenDarstellung dieses $x$ treten nur endlich viele $p$ 's und $r$ 's auf. Wegen (iv) und (ii) gibt es $q_{1}, \ldots, q_{n} \in 2$ mit $q_{1}=q, q_{i} q_{j}=\delta_{i j} q_{i}$ und $x \in \sum_{i, j=1} q_{i} B q_{j}$. Für $v_{q_{i} q_{j}}$ schreiben wir dann auch kurz $v_{i j}$. Es ist $x=\sum_{i, j=1} x_{i j}$ mit $x_{i j} \in q_{i} B q_{j}$. Wir müssen $e^{*} x^{*} x e$ in passender Form darstellen. Nun ist $x e=\sum_{i, j=1} x_{i j} e=\sum_{i=1}^{n} y_{i}$ mit $y_{i}:=\sum_{j=1}^{n} x_{i j} e \in q_{i} B q=q_{i} B q_{1}$. Setzt man

$$
z_{i}:=v_{1 i} y_{i} \in v_{1 i} q_{i} B q=v_{1 i} B q=q v_{1 i} B q \leqq q B q \quad(1 \leqq i \leqq n) .
$$

so gilt

$$
z_{i}^{*} z_{i}=y_{i}^{*} v_{1 i}^{*} v_{1 i} y_{i}=y_{i}^{*} q_{i} y_{i}=y_{i}^{*} y_{i} .
$$

Für $i \neq j$ ist

$$
y_{j}^{*} y_{i} \in\left(q_{j} B q\right)^{*}\left(q_{i} B q\right)=q B q_{j} q_{i} B q=(0) .
$$

Daher ist

$$
\begin{aligned}
e^{*} x^{*} x e & =(x e)^{*}(x e)=\left(\sum_{i=1}^{n} y_{i}\right)^{*}\left(\sum_{j=1}^{n} y_{j}\right)=\sum_{i, j=1} y_{i}^{*} y_{j} \\
& =\sum_{i=1}^{n} y_{i}^{*} y_{i}=\sum_{i=1}^{n} z_{i}^{*} z_{i} \in q B q .
\end{aligned}
$$


Da nun $f$ eine Fortsetzung von $f_{0}$ ist und $f_{0}$ positiv ist, gilt folglich $f\left(e^{*} x^{*} x e\right)$ $=f_{0}\left(\sum_{i=1}^{n} z_{i}^{*} z_{i}\right) \geqq 0$.

Satz 1. Seien $G$ eine lokalkompakte abelsche Gruppe und A eine involutive symmetrische Banachsche Algebra mit beschränkter approximierender Eins. $G$ wirke durch isometrische *-Isomorphismen auf $A,(a, x) \rightarrow a^{x}$ für $(a, x) \in A \times G$. Für jedes $a \in A$ sei $x \rightarrow a^{x}$ stetig. $G$ wirkt dann auch auf $A^{b}$, und es sei eine abgeschlossene $G$-invariante, involutive, symmetrische Unteralgebra $U$ des Zentrums von $A^{b}$ gegeben. Für jedes $u \in U$ sei $x \rightarrow u^{x}$ stetig. Das Spektrum $\hat{U}$ von $U$ sei gleich $G$, die Wirkung von $G$ auf $\hat{U}$ sei die Linkstranslation. Die Gelfand-Darstellung $u \rightarrow \hat{u} \in C_{\infty}(G)$ sei injektiv. $S(G)$ : $=\{\hat{u} ; u \in U\}$ genüge den Bedingungen 1)-4) von p. 262 in [11]; insbesondere sei die. Algebra $S_{0}(G)$ der in $S(G)$ gelegenen stetigen Funktionen mit kompaktem Träger dicht in $S(G)$ ( für die von $U$ induzierte Norm auf $S(G)$ ). Weiter habe $U$ eine ,approximieren-

de Eins für $A^{\prime \prime}, d$.h. es gebe ein Netz $\left(u_{\lambda}\right)_{\lambda \in A}$ in $U$ mit $\lim _{\lambda} u_{\lambda} f=f$ für alle $f \in A$. Unter diesen Voraussetzungen ist auch die verallgemeinerte $L^{1}$-Algebra $B:=L^{1}(G, A)$ symmetrisch.

Beweis. Wir wollen das im Lemma formulierte Kriterium für Symmetrie verwenden. Mit $A$ besitzt auch $B$ eine approximierende Eins, vgl. [8]. Weiter haben wir die Menge $2 \subseteq B^{b}$ anzugeben. Zu diesem Zweck bilden wir die verallgemeinerte $L^{1}$ Algebra $D:=L^{1}(G, U) \cong L^{1}(G, S(G))$. $D$ läßt sich kanonisch in $B^{b}$ einbetten. Wichtig für den weiteren Beweis sind die beiden folgenden Eigenschaften von $D$ :

(1) $D$ ist eine einfache Banachsche Algebra.

(2) Die „reguläre Darstellung" $\pi$ von $D$ in $\mathscr{H}:=L^{2}(G)$, gegeben durch $(\pi(f) \xi)(x)=\int_{G} f(x+y)(-y) \xi(-y) d y$, wobei $f(x+y)$ als Element von $S(G)$ angesehen wird, ist eine topologische irreduzible *-Darstellung von $D$ (siehe [9] und [11]).

Ist $v$ eine reellwertige Funktion in $S_{0}(G)$ mit $\|v\|_{2}=\left(\int_{G}|v(x)|^{2} d x\right)^{1 / 2}=1$, und bildet man dazu $q(x):=v^{x} v$, so liegt $q$ in $D$, und $\pi(q)$ ist der Projektor auf den von $v$ aufgespannten Teilraum von $\mathscr{H}$; es ist $\pi(q)=\langle-, v\rangle v . J:=\{f \in D ; \pi(f)$ ist von endlichem Rang $\}$ ist dann ein involutives, zweiseitiges, von Null verschiedenes und mithin wegen (1) dichtes Ideal in $D$. Studiert man die im Beweis von Théorème 2 in [3] wirklich verwendeten Voraussetzungen, so stellt man fest, daß die dortige Argumentation im vorliegenden Fall auch stichhaltig ist; $d$. h. bezeichnet man mit $\mathscr{H}^{\prime}$ den von Elementen der Form $\pi(f) \xi, f \in J, \xi \in \mathscr{H}$ aufgespannten Unterraum von $\mathscr{H}$, so gilt:

(3) $\mathscr{H}^{\prime}$ ist dicht in $\mathscr{H}$.

(4) $\mathscr{H}^{\prime}$ ist invariant unter $\pi(D)$.

(5) $\mathscr{H}^{\prime}$ ist der kleinste von Null verschiedene, unter $\pi(J)$ invariante Unterraum von $\mathscr{H}$.

(6) $\pi(J)$ besteht genau aus den beschränkten linearen Operatoren $T$ endlichen Ranges auf $\mathscr{H}$ mit $T(\mathscr{H}) \subset \mathscr{H}^{\prime}$ und $T^{*}(\mathscr{H}) \subseteq \mathscr{H}^{\prime}$.

Es sei nun $\mathscr{Q}$ die Menge derjenigen $q \in D$, für die $\pi(q)$ ein Projektor vom Rang 1 ist. Da $\pi$ wegen (1) injektiv ist, erkennt man an dem durch (6) vermittelten Bild von $J$ 
sofort, daß zu diesem 2 ein Satz von Elementen $\left\{v_{p q}\right\}$ mit den Eigenschaften (i)-(iv) aus dem Lemma existiert. Weiter gilt $v_{p p}=p$ für alle $p \in \mathscr{Q}$, und $J$ ist die lineare Hüle von 2 .

Wir wollen nun die Dichtheit von $\sum_{p, q \in \mathscr{Q}} p B q$ beweisen.

Es ist $\sum_{p \in \mathcal{Q}} p B=J B$ und mithin $\left(\sum_{p \in \mathscr{Q}} p B\right)^{-}=(J B)^{-}=(D B)^{-}$. Ist nun $\left(u_{\lambda}\right)_{\lambda \in A}$ eine approximierende Eins in $U$ für $A$ und $\left(e_{\mu}\right)_{\mu \in M}$ eine approximierende Eins in der gewöhnlichen Gruppenalgebra $L^{1}(G)$, so kann man wie in [8] zeigen, daß $\left(e_{\mu} u_{\lambda}\right)_{(\mu, \lambda) \in M \times A}$ eine approximierende Eins in $D$ für $B$ ist, d. h. daB $\lim _{\mu, \lambda} e_{\mu} u_{\lambda} f=f$ für alle $f \in B$ ist. Folglich ist

$$
\left[B=(D B)^{-}=(B D)^{-}=\left(\sum_{q \in Q} B q\right)^{-}=\left(\sum_{q \in Q} q B\right)^{-}\right]
$$

und dann auch $\left(\sum_{p, q \in Q} p B q\right)^{-}=B$. Mit Hilfe des Lemmas sind wir fertig, wenn wir nun noch ein $q \in \mathscr{Q}$ angeben können, für welches $q B q$ symmetrisch ist. Da wir oben explizit solche $q$ 's konstruiert haben, genügt es zu zeigen:

(7) Sei $u=u^{*} \in U, v:=\hat{u}$ liege in $S_{0}(G)$, und es gelte $\|v\|_{2}=1$. Definiert man dann $q \in \mathscr{Z} C D$ durch $q(x)=u^{x} u$, so ist $q B q$ symmetrisch.

Für das folgende seien $u, v$ und $q$ wie oben fest gewählt. Bevor wir (7) beweisen können, müssen wir einige Eigenschaften der Elemente in $q B q$ zusammenstellen. Dafür benötigen wir zunächst:

(8) Ist $h \in A$ und $\left(x \rightarrow u^{-x} u^{-x} h\right) \in L^{1}(G, A)$, so gilt $h=\int_{G} u^{-x} u^{-x} h d x$.

$\mathrm{Zu}$ (8): Da $U$ eine approximierende Eins für $A$ besitzt und $S_{0}(G)$ in $S(G)$ dicht liegt, genügt es zu zeigen, daß $w h=w \int_{G} u^{-x} u^{-x} h d x$ für alle $w \in U$ mit $\hat{w} \in S_{0}(G)$ gilt. Für solche $w$ ist obige Gleichung aber wegen $w=\int_{G} w u^{-x} u^{-x} d x \quad\left(\|v\|_{2}=1\right.$ !) offenkundig erfüllt.

(9a) Sei $f \in q B \subseteq L^{1}(G, A)$. Dann gibt es eindeutig $h \in A$ mit $f(x)=u^{x} h$ für fast alle $x \in G$. Es ist $h=\int_{G} u^{-x} f(-x) d x=\int_{G} u^{-x} u^{-x} h d x$. Gilt umgekehrt für ein $h \in A$, da $B$ $\left(x \rightarrow u^{x} h\right) \in B$, so liegt $\left(x \rightarrow u^{x} h\right)$ in $q B$.

$\mathrm{Da}(q B)^{*}=B q$, gibt es auch eine ,duale Fassung":

(9b) Sei $g \in B q$. Dann gibt es eindeutig $k \in A$ mit $g(x)=u k^{x}$ für fast alle $x \in G$. Es ist $k=\int_{G} u^{-x} g(x)^{-x} d x=\int_{G} u^{-x} u^{-x} k d x$. Gilt umgekehrt für ein $k \in A$, daB $\left(x \rightarrow u k^{x}\right) \in B$, so liegt $\left(x \rightarrow u k^{x}\right)$ in $B q$.

Zu (9a): Für fast alle $x \in G$ gilt

$$
\begin{aligned}
f(x) & =(q * f)(x)=\int_{G} q(x+y)^{-y} f(-y) d y=\int_{G}\left(u^{x+y} u\right)^{-y} f(-y) d y \\
& =\int_{G} u^{x} u^{-y} f(-y) d y=u^{x} \int_{G} u^{-y} f(-y) d y,
\end{aligned}
$$


da letzteres Integral wegen $\left\|u^{-y} f(-y)\right\| \leqq\left\|u^{-y}\right\|\|f(-y)\|=\|u\|\|f(-y)\|$ existiert. Aus (8) folgt zum einen die Eindeutigkeit von $h$ und zum anderen, daß Elemente der Form $x \rightarrow u^{x} h$ in $q B$ liegen, sofern sie nur in $B$ liegen, also integrierbar sind.

(9b) beweist man ähnlich. Indem man ausnutzt, daß die Involution in $B$ durch $f^{*}(x)=\left[f(-x)^{x}\right]^{*}$ erklärt ist, kann man auch (9b) direkt aus (9a) herleiten. (9a) und (9b) verwenden wir nun zur Bestimmung von $q B q=q B \cap B q$.

(10) Ist $f \in q B q$, so gibt es eindeutig $h \in A$ mit $u^{x} h=u h^{x}$ für alle $x \in G$ und $f(x)$ $=u^{x} h$ für fast alle $x \in G$. Ist umgekehrt $h \in A$ mit $u^{x} h=u h^{x}$ für alle $x \in G$ und liegt $\left(x \rightarrow u^{x} h\right)$ in $B$, so liegt dieses Element in $q B q$. Dieses Element ist genau dann hermitesch in $B$ bzw. in $q B q$, wenn $h=h^{*}$.

$\mathrm{Zu}(10)$ : Nach (9a) und (9b) ist $f(x)=u^{x} h$ und $f(x)=u k^{x}$ für fast alle $x \in G$ mit wohlbestimmten $h, k \in A$. Wir haben $h=k$ zu zeigen. Da $x \rightarrow u^{x} h$ und $x \rightarrow u k^{x}$ stetig sind, gilt $u^{x} h=u k^{x}$ für alle $x$; insbesondere ist $u h=u k=k u$. Nach $(9 \mathrm{a}, \mathrm{b})$ ist

$$
\begin{aligned}
k & =\int_{G} u^{-x} f(x)^{-x} d x=\int_{G} u^{-x} u h^{-x} d x=\int_{G} u(u h)^{-x} d x=\int_{G} u(k u)^{-x} d x \\
& =\int_{G} u k^{-x} u^{-x} d x=\int_{G} f(-x) u^{-x} d x=h .
\end{aligned}
$$

Es sind nun nur noch die hermite'schen Elemente in $q B q$ zu charakterisieren. Es sei $f \in q B q$ gegeben durch $f(x)=u^{x} h=u h^{x}$ für alle $x \in G$. Dann gilt $f^{*}(x)=\{f$ $\left.(-x)^{x}\right\}^{*}=\left\{\left(u h^{-x}\right)^{x}\right\}^{*}=\left\{u^{x} h\right\}^{*}=u^{x} h^{*}$ (u ist hermite'sch). Also gilt $f=f^{*}$ genau dann, wenn $h=h^{*}$.

(11) Sei $f \in q B q$ gegeben durch $f(x)=u^{x} h=u h^{x}$. Dann ist $h h^{x}=h^{x} h$ für alle $x \in G$ und folglich $h^{y} h^{x}=h^{x} h^{y}$ für alle $x, y \in G$.

$\mathrm{Zu}(11)$ : Nach (8) ist

$$
\begin{aligned}
h h^{x} & =\int_{G} u^{-y} u^{-y} h d y h^{x}=\int_{G} u^{-x} u^{-y} h h^{x} d y=\int_{G} u^{-y} u h^{-y} h^{x} d y \\
& =\int_{G} u^{-y} h^{-y} u h^{x} d y=\int_{G} u^{-y} h^{-y} u^{x} h d y=\int_{G} u^{-y} h^{x} u^{-y} h d y,
\end{aligned}
$$

da $h^{y} u^{x}=h^{x} u^{y}$ für alle $x, y \in G$.

Unter erneuter Verwendung von (8) erhält man letztlich $h h^{x}=h^{x} h$. Der Träger von $v=\hat{u}$ sei mit $K$ bezeichnet. Dann gilt $q(x)=u^{x} u=0$ für $x \notin L:=K+(-K)$ und weiter

(12) Ist $f \in q B q$ gegeben durch $f(x)=u^{x} h=u h^{x}$, so ist $h^{x} h=h h^{x}=0$ für $x \notin L$.

$\mathrm{Zu}$ (12): Für alle $x \in G$ ist

$$
u h^{x}=u^{x} h=u^{x} \int_{G} u^{-y} u^{-y} h d y=u^{x} \int_{G} u^{-y} u h^{-y} d y=\int_{G} u^{-y} u^{x} u h^{-y} d y
$$

und folglich $u h^{x}=u^{x} h=0$ für $x \notin L$. Oben, in (11), hatten wir als Zwischenergebnis $h h^{x}=\int_{G} u^{-y} h^{-y} u^{x} h d y$ für alle $x \in G$. Daraus folgt (12) nun unmittelbar.

\section{Wir kommen nun schließlich}

$\mathrm{Zu}$ (7): Es genügt zu zeigen, daß das Spektrum eines hermite'schen Elementes $f \in q B q$ reell ist. Wir haben also zu zeigen, daß die Gleichung

$$
f g=\lambda g+f
$$


für ein gegebenes $\lambda \in \mathbb{C} \backslash \mathbb{R}$ eine Lösung $g \in q B q$ besitzt. Wenn man nämlich (*) lösen kann, so besitzt auch jede Gleichung $f g^{\prime}=\lambda g^{\prime}+b$ mit $b \in q B q$ eine Lösung $g^{\prime}$ in $q B q$ und, durch Anwenden der Involution, auch jede Gleichung $g^{\prime} f=\lambda g^{\prime}+b$; also gilt $(f-\lambda) B=B(f-\lambda)=B$. Um nun $(*)$ zu lösen, setzen wir $w:=\int_{L} u^{-x} h^{-x} d x$ $=\int_{L} u^{x} h^{x} d x \in A$, wenn $f$ durch $h$ gegeben ist, wenn also $f(x)=u^{x} h=u h^{x}$ ist. Da $f$ nach Voraussetzung hermite'sch ist, ist $h=h^{*}$ nach (10) und wegen $u=u^{*}$ und der Zentralität von $u$ ist $w=w^{*}$. Laut Voraussetzung ist $A$ symmetrisch, und die Gleichung

$$
w k=\lambda k+h
$$

besitzt folglich eine eindeutig bestimmte Lösung $k \in A$. Da $x \rightarrow u^{x}$ weinen kompakten Träger hat, hat auch $x \rightarrow u^{x} w k$ einen kompakten Träger und liegt nach $(9 \mathrm{a})$ in $q B$.

Dann liegt aber auch $g$, definiert durch $g(x)=u^{x} k$, in $q B$, da $g(x)=\frac{1}{2}\left\{u^{x} w k-u^{x} h\right\}$.

Nach (11) vertauscht $h^{y}$ mit $w$ für alle $y \in G$, es gilt daher $w h^{y} k=\lambda h^{y} k+h^{y} h$ für alle $y \in G$. Nach (12) ist $h^{y} h=0$ für $y \in G \backslash L$ und mithin $h^{y} k=0$ für $y \in G \backslash L$, da $w-\lambda$ wegen der Symmetrie von $A$ ein invertierbarer Operator auf $A$ ist. Wir zeigen nun, daß $g \in q B$ eine Lösung von (*) ist.

Es gilt

$$
\begin{aligned}
(f * g)(x) & =\int_{G} f(x+y)^{-y} g(-y) d y=\int_{G}\left(u^{x+y} h\right)^{-y} u^{-y} k d y=\int_{G} u^{x} h^{-y} u^{-y} k d y \\
& =\int_{L} u^{x} h^{-y} u^{-y} k d y
\end{aligned}
$$

da $h^{-y} k=0$ für $y \in G \backslash L$.

Also gilt $(f * g)(x)=u^{x} w k=u^{x}(\lambda k+h)$ oder $f * g=\lambda g+f$. Dann ist aber $g * q$ eine Lösung von $(*)$ in $q B q$ und Satz 1 ist bewiesen.

Es ist nun recht einfach, unser Hauptergebnis zu beweisen.

Satz 2. Sei $G$ eine zusammenhängende nilpotente Liesche Gruppe. Dann ist $L^{1}(G)$ eine symmetrische Algebra.

Beweis. Wir beweisen den Satz durch Induktion über $\operatorname{dim} G$. Falls $\operatorname{dim} G=1$, so ist $G$ kommutativ und $L^{1}(G)$ bekanntlich eine kommutative symmetrische Algebra. Sei nun $\operatorname{dim} G=: n>1$. Da Quotienten symmetrischer Algebren offenkundig symmetrisch sind, kann man o.B.d.A. annehmen, daB $G$ einfach-zusammenhängend ist. Wie in [12] dargelegt, genügt es zu zeigen: Ist $\varrho$ eine beschränkte, algebraisch irreduzible Darstellung von $L^{1}(G)$ in dem Banachschen Raum $E$ und ist $P$ : $=\operatorname{Kern} \varrho \cap(\operatorname{Kern} \varrho)^{*}$, so ist $L^{1}(G) / P$ symmetrisch.

Wegen seiner Kürze sei dieses Argument hier kurz wiederholt. Wäre nämlich $L^{1}(G)$ nicht symmetrisch, so gäbe es $f \in L^{1}(G)$ mit $L^{1}(G)\left(1+f^{*} f\right) \subset L^{1}(G)$. Zu dem modularen Linksideal $L^{i}(G)\left(1+f^{*} f\right)$ gäbe es dann ein umfassendes maximales modulares Linksideal $\Lambda$ mit Rechtseins $-f^{*} f$. Man hätte dann eine beschränkte, algebraisch irreduzible Darstellung $\varrho$ von $L^{1}(G)$ in $E:=L^{1}(G) / A$, aber $L$ : 
$=L^{1}(G) / \operatorname{Kern} \varrho \cap(\operatorname{Kern} \varrho)^{*}$ wäre nicht symmetrisch, da $L\left(1+g^{*} g\right) \subsetneq L$, wenn $g$ das Bild von $f$ in $L$ bezeichnet. Seien nun $E, \varrho$ und $P$ wie oben. Wie im Falle unitärer Darstellungen stammt $\varrho$ von einer beschränkten stetigen Darstellung der Gruppe, welche ebenfalls mit $\varrho$ bezeichnet sei (vgl. [11], insb. p. 266), d. h. es gibt einen beschränkten, stark stetigen Homomorphismus $g$ von $G$ in die Gruppe der beschränkten invertierbaren Operatoren auf $E$ mit $\varrho(f)=\int_{G} f(x) \varrho(x) d x$ für $f \in L^{1}(G)$.

Da $\varrho$ beschränkt und $E$ algebraisch irreduzibel ist, gibt es einen Charakter $\gamma$ des Zentrums $Z$ von $G$ mit $\varrho(z)=\gamma(z) 1_{E}$ für $z \in Z$. $L^{1}(G) / P$ ist ein Quotient von $L^{1}(G / \operatorname{Kern} \gamma)$. Ist nun $\operatorname{dim} \operatorname{Kern} \gamma>0$, so ist nach Induktionsvoraussetzung $L^{1}(G / \operatorname{Kern} \gamma)$ und dann auch $L^{1}(G) / P$ symmetrisch. Wir können also dim Kern $\gamma=0$ annehmen; insbesondere ist $\operatorname{dann} \operatorname{dim} Z=1$. Nach Kirillows Lemma, vgl. [14], gibt es einen zweidimensionalen Normalteiler $N \supset Z$, einen Normalteiler $H$ der Codimension 1 ( $H=$ Zentralisator von $N$ in $G$ ) und ein Komplement $R$ zu $H$ derart, daß $G=R \ltimes H$ und daß $R \ltimes N$ die dreidimensionale Heisenberg-Gruppe ist. Wir dividieren nun Kern $\gamma$ aus und bezeichnen die erhaltenen Gruppen $G / \operatorname{Kern} \gamma$, $H / \operatorname{Kern} \gamma \ldots$ erneut mit $G, H, \ldots$. Für die durch $\varrho$ und $\gamma$ indizierten Homomorphismen schreiben wir ebenso wieder $\varrho$ und $y$. Dann ergibt sich die folgende Situation: $G=R \times H$ hat ein eindimensionales kompaktes Zentrum $Z$. Für $z \in Z$ gilt $\varrho(z)$ $=\gamma(z) 1_{E}$ mit einem nun treuen Charakter $\gamma$ von $Z$. Es ist die Symmetrie von $L^{1}(G) / P$ zu zeigen. Dazu sei $L^{1}(G)$, die Menge aller $f \in L^{1}(G)$ mit $f(x z)=\gamma(z) f(x)$ für (fast) alle $x \in G$ und $z \in Z$. Es sei $T_{\gamma}: L^{1}(G) \rightarrow L^{1}(G)$ definiert durch $\left(T_{\gamma} f\right)(x)=\int_{Z} \gamma(z) f(x z) d z$, wobei $d z$ hier das normierte Haarsche Maß von $Z$ bezeichnet. Offenbar ist $T_{\gamma}$ ein idempotenter Morphismus von $L^{1}(G)$ auf $B:=L^{1}(G)_{\gamma}$, und an der Integraldarstellung von $\varrho, \varrho(f)=\int_{G} f(x) \varrho(x) d x$, erkennt man leicht, da $\varrho \varrho=\varrho \circ T_{\gamma}$. Also ist $L^{1}(G) / P$ ein Quotient von $L^{1}(G) / \operatorname{Kern} T_{\gamma} \cong B$, und es genügt, die Symmetrie dieser Algebra zu beweisen.

Analog zu $L^{1}(G)_{\gamma}$ kann man auch die Algebren $A:=L^{1}(H)_{\gamma} \subseteq L^{1}(H)$ und $U$ : $=L^{1}(N)_{\gamma} \subseteq L^{1}(N)$ bilden. $L^{1}(G)$ fassen wir nun als verallgemeinerte $L^{1}$-Algebra $L^{1}\left(R, L^{1}(H)\right)$ auf mit trivialem Faktorensystem und der durch die zu $R$ gehörigen inneren Automorphismen induzierten Wirkung von $R$ auf $L^{1}(H)$. Offenkundig ist $B$ dann gerade die Unteralgebra $L^{1}(R, A)$. $U$ ist isometrisch *-isomorph zu $L^{1}(\mathbb{R})$ und hat mithin das Spektrum $\mathbb{R}=R$. Ferner ist $U$ als zentrale Unteralgebra von $L^{1}(H)^{b}$ (= Maßalgebra von $H$ ) und dann auch von $A^{b}$ auffaßbar. $A$ ist als abgeschlossene *-Unteralgebra von $L^{1}(H)$ nach Induktionsvoraussetzung symmetrisch. Man überlegt sich leicht, daß auch die übrigen Voraussetzungen von Satz 1 in der vorliegenden speziellen Situation erfüllt sind. $B$ ist folglich symmetrisch, und Satz 2 ist bewiesen.

Wir schließen mit der folgenden

Bemerkung. Aus Satz 2 ergibt sich mit Hilfe von (6) aus [12], daß für eine zusammenhängende nilpotente Liesche Gruppe $G$ die Menge der Kerne topologisch irreduzibler *-Darstellungen von $L^{1}(G)$ mit der Menge der primitiven Ideale (im Sinne der Algebra) und mit der Menge der maximalen abgeschlossenen zweiseitigen Ideale übereinstimmt. 


\section{Literatur}

1. Anusiak, Z.: Symmetry of $L^{1}$-group algebras of locally compact groups with relatively compact classes of conjugated elements. Bull. Acad. Pol. Sci. 18, 329-332 (1970)

2. Bonsall,F.F., Duncan, 1.: Complete normed algebras. Ergebnisse der Mathematik 80 . BerlinHeidelberg-New York: Springer 1973

3. Dixmier,J.: Opérateurs de rang fini dans les représentations unitaires. Publ. math. Inst. Hautes Etudes scient. 6, 305-317 (1960)

4. Gangolli, R.: On the symmetry of $L^{1}$-algebras of locally compact motion groups and the Wiener Tauberian theorem. Preprint Seattle (1975)

5. Hulanicki, A.: On symmetry of group algebras of discrete nilpotent groups. Studia Math. 35, 207-219 (1970)

6. Jenkins, J.: Nonsymmetric group algebras. Studia Math. 45, 295-307 (1973)

7. Leptin, $H$.: Verallgemeinerte $L^{1}$-Algebren und projektive Darstellungen lokal kompakter Gruppen. I. Inventiones math. 3, 257-281 (1967)

8. Leptin, H. Darstellungen verallgemeinerter $L^{1}$-Algebren. Inventiones math 5, 192--215 (1968)

9. Leptin, H.: On group algebras of nilpotent Lie groups. Studia Math. 47, 37-49 (1973)

10. Leptin, H.: Harmonische Analyse auf gewissen nilpotenten Lieschen Gruppen. Studia Math. 48, $201-205(1973)$

11. Leptin, H. Ideal theory in group algebras of locally compact groups. Inventiones math. 31, 259-$278(1976)$

12. Leptin, H.: Symmetrie in Banachschen Algebren. Erscheint im Archiv der Mathematik

13. Leptin, H. : Lokal kompakte Gruppen mit symmetrischen Algebren. Vorabdruck, Bielefeld (1976)

14. Pukanszky,L.: Leçons sur les représentations des groupes. Paris: Dunod 1967

Eingegangen am 5. Juli 1976 